\title{
Sub-wavelength Uniform Periodic Surface Structures Induced by Low Femtosecond Laser Fluence on Silicon with Optical Glass Covering
}

\author{
Chao Dong, NingYi Yuan**, JianhuaQiu, Biao Kan, JianNing Ding** \\ 1. Jiangsu Collaborative Innovation Center of Photovolatic Science and Engineering, Changzhou \\ UUniversity, Changzhou, 213164, Jiangsu, China \\ 2. Jiangsu Key Laboratory for Photovolatic Engineering and Science, Changzhou University, \\ Changzhou, 213164, Jiangsu, China \\ 3. Center for Low-Dimensional Materials, Micro-Nano Devices and Systems, Changzhou University, \\ Changzhou, 213164, Jiangsu, China \\ Correspondingauthor: nyyuan@cczu.edu.cn,dingjn@cczu.edu.cn
}

\begin{abstract}
Thus far, femtosecond laser-induced periodic surface structures (LIPSS) on the surfaces of solid materials have been studied in air and liquids. However, few studies have investigated LIPSS formation on a solid surface through a solid transparent medium material. In the present study, LIPSS are formed on a silicon wafer surface covered with optical glass (glass environment) and compared with those formed in air. The periodicities of low and high spatial frequency LIPSS (LSFL and HSFL, respectively) formed in the two environments are 650 and $200 \mathrm{~nm}$, respectively. In glass environment, LSFL can be induced by laser fluence of $410 \mathrm{~mJ} / \mathrm{cm}^{2}$, which is much lower than that of $570 \mathrm{~J} / \mathrm{cm}^{2}$ used in air. Moreover, LIPSS formed in glass environment are rough, contradicting a previous report on LIPSS formation in liquid environments. The possible mechanisms forming the different structures in the two environments are discussed.

DOI: $10.2961 / \mathrm{jlmn} .2015 .02 .0015$
\end{abstract}

Keywords: periodic surface structures, low femtosecond laser fluence, silicon, optical glass

\section{Introduction}

Laser-induced periodic surface structures (LIPSS) were first experimentally observed by Brinbaum in 1965 [1]. Since then, they have been studied on various solid materials such as metals, semiconductors, insulators, and alloys in air [2-7]. Upon irradiation using a linearly polarized femtosecond laser pulse, the formation of two distinct types of LIPSS-low and high spatial frequency LIPSS (LSFL and HSFL, respectively)-has been observed. The spatial periodicity of LSFL is usually shorter than the laser wavelength owing to interference between the incident laser beam and the surface-scattered light [8], nonlinear energy absorption and non-equilibrium thermodynamics process [9-11], local field enhancement $[12,13]$, or surface plasmon modes [1315]. Furthermore, the spatial periodicity of HSFL is much shorter than that of LSFL. Irradiation using a laser with energy near the threshold involved in the formation of LIPSS structures has many applications including switching transistors [16], MEMS [17], and photovoltaics [18].

Apart from air, many researchers have produced femtosecond LIPSS on solid materials in liquids because liquids can provide a better heat sink, cool the sample surfaces efficiently, and reduce laser-induced residual thermal damages. Albu et al. [19] reported the formation of LIPSS on metallic films $(\mathrm{Cr}, \mathrm{Ti}$, and $\mathrm{W})$ in water $\left(\mathrm{H}_{2} \mathrm{O}\right)$, ethanol $\left(\mathrm{C}_{2} \mathrm{H}_{5} \mathrm{OH}\right)$, and chloroform $\left(\mathrm{CHCl}_{3}\right)$. They observed LSFL and HSFL with periodicities of 350-600 and 50-200 nm, respectively; furthermore, the periods of the structures formed in liquids were several times smaller than those of the structures formed in air. Liu et al. [20] systematically investigated the interaction of a femtosecond laser pulse with silicon in water, alcohol, and air. They found that the threshold fluence of Si was reduced by the presence of liquids (water/alcohol) and that the ablation depths of craters were deepened in water environment. SEM images showed that LIPSS formed in liquid environments were smooth because liquids could remove ejections. Thus far, studies on LIPSS have mainly focused on the surfaces of solid materials in air or liquids [19,20]; in these studies, laser irradiation occurred at the air/solid or liquid/solid interface. However, to our knowledge, there are no investigations about laser irradiation at the transparent-solid/solid interface.

In the present study, we investigate periodic surface structures induced by femtosecond laser irradiation on a silicon wafer in air and glass environments. In the latter case, an optical glass sheet was used to cover the silicon wafer surface tightly to ensure that no air bubbles remained entrapped at the glass/silicon interface. The morphological characteristics of optical glass and silicon were investigated by field-emission scanning electron microscopy (FE-SEM, Hitachi, Japan), and atomic force microscopy (AFM, Bruker, USA).

\section{Experimental section}

In our experiments, we used a 300- $\mu$ m-thick singlecrystal polished silicon (100) wafer and 1700- $\mu$ m-thick optical glass sheet as the sample. The root-mean-square surface roughness $\left(d_{\mathrm{RMS}}\right)$ values of the optical glass and silicon wafer were $\sim 0.26 \mathrm{~nm}$ and $0.29 \mathrm{~nm}$, respectively. Before the experiments, both the Si wafer and the optical glass were cleaned in an ultrasonic bath for $15 \mathrm{~min}$ in acetone and rinsed in alcohol. 
The optical glass sheet and silicon wafer were put together tightly. Then, the sample was illuminated by infrared light and CCD was used to detect the transmission signal to observe if there were any air bubbles entrapped at the glass/silicon interface [21]. If there were, interference would be generated due to different optical path and formed the Newton ring. In this work, the transmission signal showed no obvious defects, revealed that no air bubbles were entrapped at the glass/silicon interface.

A Ti:sapphire oscillator amplifier (Coherent, USA) was used as the femtosecond laser source. The well-defined Gaussian-shaped output beam has a laser pulse wavelength of $800 \mathrm{~nm}$ with pulse duration of $35 \mathrm{fs}$. Its maximum pulse energy is $4 \mathrm{~mJ}$ and repetition rate is $1 \mathrm{kHz}$. The laser beam was adjusted using a combination of a half-wave plate and a linear polarizer and then focused on the sample surface through a lens with a focal length of $25 \mathrm{~cm}$. The sample was mounted on a computer-controlled three-axis (X-Y-Z) stage (PI, USA). The fluences were estimated hereinafter by using the relation $F=2 E_{p} / \pi \omega_{0}^{2}=2 E_{p} /\left(\pi D^{2} / 4\right)$, where $E_{p}$ is the average energy per pulse and $D \approx 25 \mu \mathrm{m}$ is the Gaussian beam diameter at $1 / \mathrm{e}^{2}$ at the surface of the sample measured with a CCD camera. Throughout the laser experiments, the fluence of silicon surface was $F=300-600$ $\mathrm{mJ} / \mathrm{cm}^{2}$, corresponding to $E_{p}=0.7-1.4 \mu \mathrm{J}$. This fluence was similar to the ablation threshold $F_{t h}=400-500 \mathrm{~mJ} / \mathrm{cm}^{2}$ and much higher than the melting or modification threshold $F_{m}$ $=260-350 \mathrm{~mJ} / \mathrm{cm}^{2}$ of crystalline $\mathrm{Si}$ for a single fs laser pulse at $\lambda \sim 800 \mathrm{~nm}$ in air $[22,23]$. Fig. 1 shows a schematic diagram of the experimental setup.

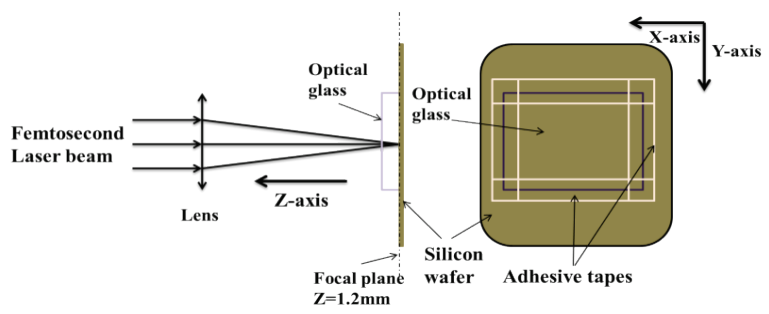

Fig.1 The optical path used in glass environment and assembly drawing of sample

The focal planes in air and glass environments were adjusted along the optical axis (Z-axis) to $\mathrm{z}=0$ and $\mathrm{z}=1.2$ $\mathrm{mm}$, respectively. Laser fluence of 570,410 , and 300 $\mathrm{mJ} / \mathrm{cm}^{2}$ and writing speed of $1 \mathrm{~mm} / \mathrm{s}$ were used. The writing direction of the laser beam was parallel to the laser polarization direction. After laser irradiation, the optical glass covering the silicon wafer was removed. Then, the sample was treated in an ultrasonic bath for $5 \mathrm{~min}$ in both deionized water and acetone to clean the residual ejections of material off the surface.

\section{Results and discussion}

The pulse number of the laser in per unit area could be calculated using the equation $N=2 r_{0} f / v$, where $r_{0}(\mu \mathrm{m})$ is the beam radius at the focal plane; $f(\mathrm{~Hz})$, the frequency of the laser pulse; and $v(\mu \mathrm{m} / \mathrm{s})$, the moving speed of the sample. So, in the case of our experiment, the pulse number of laser in per unit area was almost 25. Fig. 2 shows FESEM images of the structures formed on the silicon wafer sur- face at different laser pulse energies in the two environments. In air environment, as shown in Fig. 2 ( $a, b$, and c), for a laser fluence of $570 \mathrm{~mJ} / \mathrm{cm}^{2}$, relatively uniform LIPSS with periodicity of $650 \mathrm{~nm}$ were formed [(Fig.2 (a)]. However, some etched pits appeared in the center region mainly because the Gaussian laser beam used has relatively high energy in this region. At pulse energies of 410 and 300 $\mathrm{mJ} / \mathrm{cm}^{2}$, similar LIPSS but with incomplete structures and reduced average depth were formed on the silicon wafer surface. In glass environment, as shown in Fig.2 (e), for a laser pulse energy of $410 \mathrm{~mJ} / \mathrm{cm}^{2}$, which was much lower than that in air environment, uniform LIPSS were formed. At laser fluence of $570 \mathrm{~mJ} / \mathrm{cm}^{2}$, LIPSS were formed only in the periphery of the laser-treated areas whereas the silicon surface was damaged in the center region [Fig.2 (d)]. Fig.2 (f) and the magnified view in Fig.2 (g) show that HSFL with a periodicity of $300 \mathrm{~nm}$ were formed in the periphery of areas treated with a much smaller laser fluence. In two experiments, the orientations of two types of LIPSS were both perpendicular to the laser polarization direction. SEM images of areas treated with the same pulse energy showed that structures formed in the glass environment were rougher than those formed in air, which contradicted a previous report [20] that structures formed in liquids environments were smooth. This is mainly because the confinement of glass, most of the ejections will deposit on the silicon surface during laser experiment immediately. In contrast, ejections will be removed by airflow in air environment, and bubbles formed during laser treatment in liquid environment. Seen from the Fig. 2, the ejection particles si-

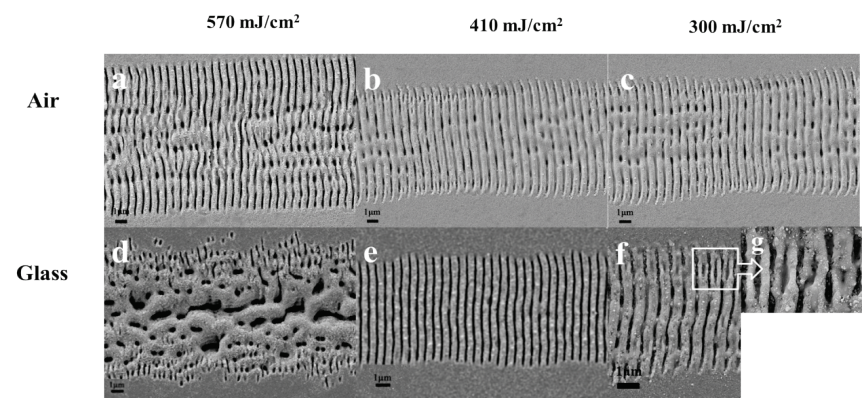

Fig.2 FESEM images of the laser treated Si area with the different laser pulse energy when the scanning speed is $1 \mathrm{~mm} / \mathrm{s}$ in two different environments. Inset $(\mathrm{g})$, the magnification image of HSFL

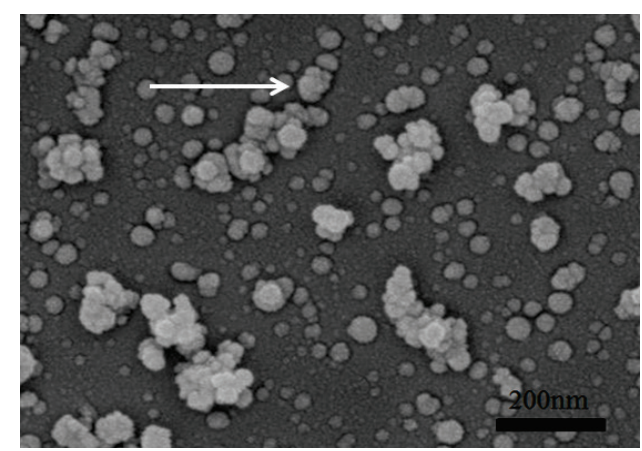

Fig.3 FESEM image of the glass surface after femtosecond laser treatment with the fluence of $410 \mathrm{~mJ} / \mathrm{cm}^{2}$. (The arrow towards the direction of laser pulse writing.)

zes deposited on the silicon surface were almost in the range of 10-100 nm. Such ejections would not affect the 
subsequent laser pulse with the beam diameter of $25 \mu \mathrm{m}$. Fig. 3 is the FESEM image of glass surface after laser treatment with the fluence of $410 \mathrm{~mJ} / \mathrm{cm}^{2}$, which shows a few ejections deposited on the glass surface.

Fig. 4 shows AFM images and sectional view of the uniform LSFL treated by a femtosecond laser in both environments. From the sectional view, the average depth of the LSFL formed in glass environment with laser fluence of $410 \mathrm{~mJ} / \mathrm{cm}^{2}$ is $320 \mathrm{~nm}$, which is approximately equal to the structure depth of $300 \mathrm{~nm}$ formed in air environment with laser fluence of $570 \mathrm{~mJ} / \mathrm{cm}^{2}$. The modulation depth produced in glass environment is much deeper than the one previously reported [19]. The AFM images showed that LIPSS formed in glass environment were uniform mainly because glass can confine ejections to provide uniform conditions on the silicon surfaces during laser beam irradiation. In air and liquid environments, ejections or bubbles would distribute around the silicon wafer surface and subsequently interact with the incident laser beam. Then, scattering, absorption, and other processes would create nonuniform conditions on the silicon wafer surfaces, leading, finally, to the formation of nonuniform structures on the silicon surfaces.
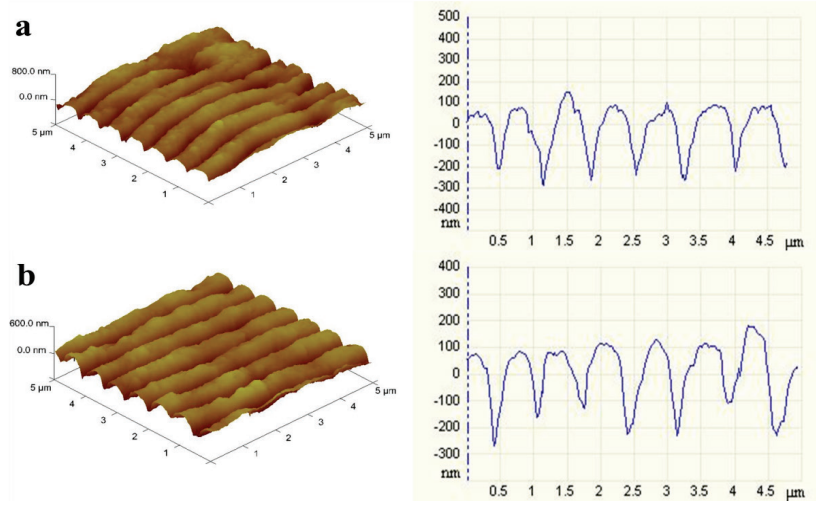

Fig.4 AFM images and sectional view of LIPSS: (a) in air environment with laser fluence of $570 \mathrm{~mJ} / \mathrm{cm}^{2}$; (b) in glass environment with laser fluence of $410 \mathrm{~mJ} / \mathrm{cm}^{2}$

The reduction in laser fluence when forming LIPSS in a glass environment could be explained by the energy loss caused by interfacial reflection as given by the following equation: $R=\left(\left(n-n_{0}\right)^{2}+k^{2}\right) /\left(\left(n+n_{0}\right)^{2}+k^{2}\right)$, where $R$ is the reflection coefficient; $n$ and $k$ are the real and imaginary parts of the refractive index of the target, respectively; and $n_{0}$ is the refractive index of air. As reported previously [20], a laser-treated silicon wafer in liquid environments could reduce the ablation threshold fluence owing to the different optical properties at the interfaces of transparent mediums and the silicon wafer. The reflection-induced energy loss in water and alcohol is $9 \%$ and $10 \%$ lower than that in air, respectively. In our experiments, the laser would be reflected at the air/glass and glass/silicon interfaces. Specifically, in air environment, the laser would be reflected at the air/glass interface; while in glass environment, the laser would be reflected at the air/glass and glass/silicon interfaces. At the air/glass interface, considering $k=0$ for glass, $R$ is calculated to be $4.3 \times 10^{-2}$, and at medium/silicon interfaces, considering $\mathrm{n}$ and $k$ for silicon $(\lambda=800 \mathrm{~nm}, 300 \mathrm{~K})$ to be 3.42 and 0.004 , respectively, $R$ is calculated to be
$3.00 \times 10^{-1}$ and $1.46 \times 10^{-1}$ for air and glass, respectively. Therefore, for the same incident laser fluence, the reflection-induced energy loss in ambient air is $11 \%$ higher than that in the glass environment. Therefore, more energy can be injected into the sample surfaces in glass environment.

Formation of sub-wavelength LIPSS on silicon could be well explained by the interference of laser light with SPPs [15]. Due to the experimental condition of $F>F_{t h}$, for crystal-Si (c-Si), the superimposed shots of fs laser pulse will form a thin amorphous-Si (a-Si) layer of a few tens of nanometers in thickness $[22,23]$ in which incident laser pulse will predominantly produces a high density of free electrons $\mathrm{Ne}$; the optical properties of a-Si layer should be determined by abundant hot electrons rather than the c$\mathrm{Si}$. Then, the SPPs can transiently be excited via the coherent coupling of the incident laser pulse with the randomly corrugated surface, where the a-Si layer including $\mathrm{Ne}$ works as a thin metal layer between glass and the c-Si substrate for the excitation of SPPs [24]. This excited material layer including Ne works as a thin metal layer and supports the formation of SPPs which in turn undergo interference with the laser light leading to the formation of subwavelength features [24]. SPPs can be excited when the familiar dispersion relation $\lambda_{s}=\lambda_{0} \sqrt{\left(\varepsilon^{\prime}+\varepsilon_{d}\right) / \varepsilon^{\prime} \varepsilon_{d}}$ is satisfied [24], where $\lambda_{0}$ and $\lambda_{\mathrm{s}}$ are the wavelength of incident laser and surface plasmons, $\varepsilon$ ' is the real part of the dielectric constant of a-Si, and $\varepsilon_{d}$ is the dielectric constant of glass. Spatial period $(\Lambda)$ of the ripples formed by the interference of laser with SPPs is expressed as $\Lambda=\lambda_{0} /\left(\lambda_{0}\right)$ $\left.\lambda_{s} \pm \sin \theta\right)$. In the present experiments, laser is incident normally (i.e., $\theta=0^{\circ}$ ) on the silicon surface, therefore, the spatial period can be expressed as $\Lambda=\lambda_{s}=\lambda_{0} \sqrt{\left(\varepsilon^{\prime}+\varepsilon_{d}\right) / \varepsilon^{\prime} \varepsilon_{d}}$. In the calculation of $\lambda_{0}=800 \mathrm{~nm}$, we used $\varepsilon^{\prime}=14.9+i 0.627$ [25] and $\varepsilon_{d}=1.1-2.2$ [26], the calculation periodicities are $\Lambda=580-790 \mathrm{~nm}$, which is in good agreement with the observed uniform periods of $650 \mathrm{~nm}$.

\section{Conclusions}

The laser fluences of LIPSS formed in glass and air environments were 410 and $570 \mathrm{~mJ} / \mathrm{cm}^{2}$. The lower laser fluence in glass environment was attributable to the decreased reflectivity at the glass/silicon and air/glass interfaces with the incident laser pulses energies. The modulation depth produced in glass environment is much deeper than the one previously reported. Moreover, laser writing speed used in experiments is $1 \mathrm{~mm} / \mathrm{s}$, faster than many previously reported, indicated fewer laser pulses exit in per unit area. This work provides an efficient way to produced large uniform LIPSS on advanced electro-optic devices. The formation mechanism of LSFL in the two environments was explained by the SPPs models.

\section{Acknowledgments}

This work was supported by National High Technology Research and Development Program 863 (2011AA050511), The National Natural Science Foundation of China (51272033), and Jiangsu “333” Project (201041).

\section{References}


[1] M. Birnbaum: J. Appl. Phys., 36, (1965) 3688-3689.

[2] F. Costache, M. Henyk, J. Reif: Appl. Surf. Sci., 186, (2002) 352-357.

[3] E.L. Gurevich: Phys. Rev. E., 83 (2011) 031604.

[4] M. Straub, M. Afshar, D. Feili, H. Seidel, K. König: Phys. Procedira 12 (2011) 16-23.

[5] E. Rebollar, S. Pérez, J.J. Hernández, I. Martín-Fabiani, D.R. Rueda, T.A. Ezquerra, M. Castillejo: Langmuir., 27, (2011) 5596-5606.

[6] J. Bonse, A. Rosenfeld, J. Krüger: J. Appl. Phys., 106, (2009) 104910.

[7] E.L. Gurevich: Appl. Surf. Sci., 278, (2013) 52-56.

[8] A. Siegman, P. Fauchet: IEEE J. Quantum Elect., 22, (1986) 1384-1403.

[9] S.K. Sundaram, E. Mazur: Nat. Mater., 1, (2002) $217-$ 224.

[10] A. Rousse, C. Rischel, S. Fourmaux, I. Uschmann, S. Sebban, G. Grillon, P. Balcou, E.Forster, J.P. Geindre, P. Audebert, J.C. Gauthier, D. Hulin: Nature, 410, (2001) 65-68

[11] K. Sokolowski-Tinten, J. Bialkowski, D. von der Linde: Phys. Rev. B (Condens Matter), 51, (1995) 14186-14198.

[12] H. Min, Z. Fuli, C. Ya, X. Ningsheg, X. Zhizhan: Phys. Rev. B (Condens Matter Mater Phys), 79, (2009) 125436.

[13] G. Miyaji, K. Miyazaki: Opt. Express, 16, (2008) 16265-16271.

[14] S. Sakabe, M. Hashida, S. Tokita, S. Namba, K. Okamuro: Phys. Rev. B (Condens Matter Mater Phy)., 79, (2009) 033409.
[15] M. Huang, F. Zhao, Y. Cheng, N. Xu, Z. Xu: ACS Nano., 3, (2009) 4062-4070.

[16] Y. Sugawara, Y. Uraoka, H. Yano, T. Hatayama, T. Fuyuki, A. Mimura: IEEE Electron Dev. Lett., 28, (2007) 395-397.

[17] X.Y. Chen, Y.F. Lu, B.J. Cho, Y.P. Zeng, J.N. Zeng, Y.H. Wu: Appl. Phys. Lett., 81, (2002) 1344-1346.

[18] B.K. Nayak, V.V. Iyengar, M.C. Gupta: Progress in Photovoltaics: Research and Applications 19, (2011) 631-639.

[19]C. Albu, A. Dinescu: Appl. Surf. Sci., 278, (2013) 347-351.

[20]H.W. Liu, F. Chen: Thin Solid Films, 518, (2010) 5188-5194.

[21] L. Di Cioccio, F. Letertre, Y. Le Tiec, A.M. Papon, C. Jaussaud, M. Bruel: Mater. Sci. Forum, 264, (1998) 765-770

[22] J. Bonse, K.-W. Brzezinka, and A. J. Meixner: Appl. Surf. Sci., 221(1-4), (2004) 215-230.

[23] Y. Izawa, Y. Izawa, Y. Setsuhara, M. Hashida, M. Fujita, R. Sasaki, H. Nagai, and M. Yoshida: Appl. Phys. Lett., 90(4), (2007) 044107.

[24] H. Raether: "Surface Plasmons on Smooth and Rough Surfaces and on Gratings" ed. by H. Raether, (Springer, Berlin, 1988) p.756.

[25] G. Miyaji, K. Miyazaki: Opt. Express, 20, (2012) 14848.

[26] E. D. Palik: "Handbook of Optical Constants of Solids" ed. by E. D. Palik, (Academic, London, 1997) p.571.

(Received: January 16, 2015, Accepted: March 11, 2015) 